\title{
Lesão no Segmento Proximal da Artéria Coronária Descendente Anterior: Resultados entre o Tratamento Cirúrgico (Mamária) e o Percutâneo (Stent com Fármaco)
}

\author{
Wilson Albino Pimentel Filho ${ }^{1,2}$, Maéve de Barros Correia', Edson Alcides Bocchi ${ }^{1}$, Tiago Di Nucci ${ }^{1}$, \\ Walter Beneduzzi Fiorotto ${ }^{2}$, Leonardo Martins Barroso ${ }^{2}$, Evandro Gomes de Mattos Jr. ${ }^{1}$, \\ Wellington Borges Custódio², Jorge Roberto Büchler², Stoessel de Assis Figueredoº ${ }^{2}$ Egas Armelin²
}

\section{RESUMO}

Objetivo: Correlacionar a permeabilidade e a evolução clínica de pacientes uni ou multiarteriais, portadores de doença coronária aterosclerótica obstrutiva no terço proximal da artéria coronária descendente anterior (DA), que foram submetidos a cirurgia de revascularização miocárdica utilizando a mamária interna esquerda (MIE) ou que receberam stent com eluição de fármaco (SEF). Método: Este é um estudo de coorte histórico, com a participação de dois centros. Foram analisados, retrospectivamente, 300 pacientes, nos quais foi tratada lesão no terço proximal da DA: no Grupo 1 (G-1), 150 pacientes foram submetidos a cirurgia de revascularização miocárdica e no G-2, 150 receberam SEF, 95 desses stents de paclitaxel e 55 de sirolimo. Os principais eventos cardiovasculares adversos maiores (ECAM) foram registrados no período entre ambos os procedimentos e o reestudo angiográfico. O óbito não foi incluído nesses ECAM, já que todos os pacientes foram reestudados pela coronariografia. Ocorreram diferenças demográficas clínicas e angiográficas entre os dois grupos: os pacientes do G-1 apresentavam maior incidência de diabetes $(29,3 \%$ vs. $20,0 \%$; p < 0,05$)$ e de doença aterosclerótica triarterial $(50,0 \%$ vs. $30,6 \% ; p<0,05)$, com menor incidência de uniarteriais $(13,3 \%$ vs. 30,6\%; p < 0,001$)$. Resultados: A permeabilidade da DA foi bastante alta, em ambos os grupos. Os pacientes livres de ECAM no G-1 e no G-2 ficaram assim distribuídos: em 12 meses, 98\% vs. 97\% (NS); em 24 meses, 93\% vs. 94\% (NS); e em 32 meses, $89 \%$ vs. $91 \%$ (NS). Conclusão: Ambas as técnicas de revascularização miocárdica para tratar a DA apresen-

\section{SUMMARY}

Proximal Left Anterior Descending Artery Lesion: Results Between Surgical (Mammary) and Percutaneous (Drug Eluting Stent) Treatments

Objectives: To correlate the permeability and clinical evolution of single and multilateral patients, bearers of obstructive atherosclerosis heart disease in the proximal third of the anterior descending coronary artery (DA), who were submitted to myocardial revascularization using the left internal mammary or underwent a drug eluting stent procedure. Method: This a case history cohort study in which two centers participated. Three hundred patients were retrospectively analyzed, whose lesions were treated at the proximal one third of the DA: in the G-1 group, 150 patients, underwent myocardial revascularization surgery and, the G-2 group, 150 patients, received drug eluting stents, 95 of them received paclitaxel and 55 received sirolimus. All major adverse cardiovascular events were registered during the period (MACE) during the period between the two procedures and the angiographic restudy. Deaths were not included in these MACE, since all patients were restudied through coronariography. There were clinical and angiographic demographic differences between the two groups: the G-1 patients showed a higher incidence of diabetes $(29.3 \%$ versus $20.0 \%, \mathrm{p}<0.05)$ and three artery atherosclerosis disease $50 \%$ versus $30.6 \%, \mathrm{p}<0.05)$ with a lower incidence of one artery $(13.3 \%$ versus $30.6 \%, p<0.001)$. Results: The permeability of the two procedures was quite high in both groups. Those patients free of MACE in G-1

\footnotetext{
Instituto do Coração de Campinas - Campinas, SP.

2 Real e Benemérita Sociedade Portuguesa de Beneficência de São Paulo - São Paulo, SP.

Trabalho apresentado no XXIX Congresso da Sociedade Brasileira de Hemodinâmica e Cardiologia Intervencionista - Brasília, DF - 19 a 22 de junho de 2007. Premiação de melhor pôster.

Correspondência: Wilson Albino Pimentel Filho. Rua Inhambu, 917, apto. 91 - Moema - São Paulo, SP - CEP 04529-013

E-mail: wilpm@uol.com.br

Recebido em: 23/7/2007 • Aceito em: 13/9/2007
} 
Pimentel Filho WA, et al. Lesão no Segmento Proximal da Artéria Coronária Descendente Anterior: Resultados entre o Tratamento Cirúrgico (Mamária) e o Percutâneo (Stent com Fármaco). Rev Bras Cardiol Invas 2007; 15(4): 370-377.

taram excelentes resultados, tanto na permeabilidade como na evolução clínica dos pacientes.

DESCRITORES: Revascularização miocárdica. Contenedores. Sirolimo. Paclitaxel. Implantes de medicamento.

D ados históricos ${ }^{1,2}$ têm demonstrado o efeito positivo do implante da artéria mamária interna esquerda (MIE) na artéria coronária descendente anterior (ACDA), com permeabilidade em longo prazo desse enxerto arterial e, conseqüentemente, com impacto favorável na evolução clínica tardia.

A ACDA é um "divisor de águas" quando se pretende revascularizar o paciente com doença coronária arterial obstrutiva (DCAO), principalmente naquele com acometimento multiarterial e, com maior impacto, no portador de diabetes do tipo 2, seja naquele tratado com medicação oral seja no insulino-dependente, em quem, na maioria das vezes, se privilegia o tratamento cirúrgico $^{3-5}$. Portanto, a cirurgia cardíaca utilizando a MIE para revascularizar a ACDA no paciente uni ou multiarterial faz, com certeza, a diferença na evolução clínica e, até então, tem sido o padrão de referência de revascularização da ACDA.

Por outro lado, os stents com eluição de fármacos (SEF) têm demonstrado redução significativa da reestenose e da necessidade de nova intervenção no vasoalvo quando comparados ao stent convencional, tendo, assim, evolução clínica mais favorável ${ }^{6-9}$.

No tocante ao tratamento da ACDA utilizando os SEF, tanto nos pacientes uni como nos multiarteriais, relatos contemporâneos têm demonstrado resultados alentadores, por vezes comparáveis aos achados cirúrgicos com o uso da $\mathrm{MIE}^{10-13}$.

Nesse contexto, a finalidade deste estudo foi analisar e comparar os resultados relacionados à permeabilidade, por meio do reestudo angiográfico, e à condição clínica, pela ocorrência dos eventos cardiovasculares maiores (ECAM), ambos a longo prazo, dos pacientes portadores de lesão obstrutiva grave no terço proximal da ACDA submetidos a tratamento cirúrgico utilizando MIE ou a implante de SEF.

\section{MÉTODO}

Este é um estudo de coorte histórico e com participação de dois centros.

Foram analisadas 300 coronariografias, das quais 150 eram de pacientes submetidos a cirurgia de revascularização miocárdica (CRM), denominados de Gru- and G-2 were distributed in the following way: in 12 months, $98 \%$ versus $97 \%$ (NS); in 24 months, 93\% versus $94 \%$ (NS); and in 32 months, $89 \%$ versus $91 \%$ (NS). Conclusion: Both myocardial revascularization techniques for the treatment of DA showed excellent results, in permeability as well as clinical evolution of the patients.

DESCRIPTORS: Myocardial revascularization. Stents. Sirolimus. Paclitaxel. Drug implants.

po 1 (G-1), e 150 pacientes nos quais se implantou SEF (G-2), sendo 95 com stents revestidos com paclitaxel e 55 com sirolimo. As análises das angiografias coronárias, tanto as prévias a ambos os procedimentos (cirúrgico e percutâneo) como as de revisão, foram realizadas por dois cardiologistas intervencionistas experientes. Houve, também, contato, direto ou por telefone, com o cardiologista responsável pelo paciente ou interrogatório deste, logo após o estudo coronariográfico, com a finalidade de coletar os principais ECAM que porventura ocorreram no período compreendido entre os procedimentos de revascularização e o reestudo angiográfico, assim discriminados: retorno da angina e/ou teste provocativo de isquemia positivo, novo procedimento (cirúrgico ou percutâneo), infarto do miocárdio e acidente vascular cerebral. O óbito foi excluído dos ECAM em decorrência de sua característica estrutural, ou seja, todos os pacientes foram submetidos a reestudo coronariográfico.

O tempo entre a cirurgia e o reestudo foi de 12 a 32 meses $(25 \pm 13)$ vs. a intervenção percutânea, que foi de 12 a 32 meses $(26 \pm 12)$, distribuídos de acordo com a Figura 1.

Os aspectos demográficos principais de ambos os grupos, tanto clínicos como anatômicos, por ocasião da indicação do procedimento cirúrgico ou percutâneo, estão distribuídos na Tabela 1. Nesta análise, foram excluídos pacientes com cirurgia ou angioplastia prévias e

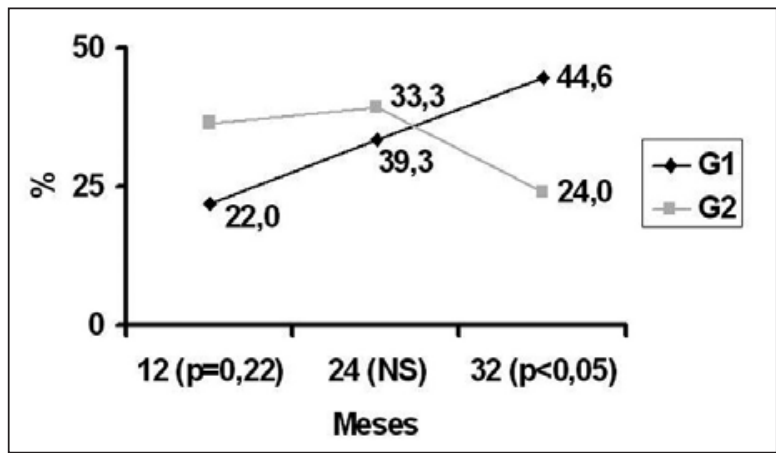

Figura 1 - Distribuição dos casos (CD, fitas ou filmes de 35 mm todos denominados de filmes) analisados de ambos os grupos, de acordo com o período entre o procedimento de revascularização e a coronariografia de revisão. 
Pimentel Filho WA, et al. Lesão no Segmento Proximal da Artéria Coronária Descendente Anterior: Resultados entre o Tratamento Cirúrgico (Mamária) e o Percutâneo (Stent com Fármaco). Rev Bras Cardiol Invas 2007; 15(4): 370-377.

infarto agudo do miocárdio com supradesnivelamento do segmento ST. A média de idade dos pacientes era semelhante entre os grupos (de 67 anos no G-1 e de 66 anos no G-2), sendo a maioria do sexo masculino (Tabela 1). Ocorreram diferenças demográficas significativas: maior contingente de pacientes diabéticos e multiarteriais no G-1 e de uniarteriais no G-2 (Tabela 1).

Foram realizadas, em média, 3,2 $\pm 2,8$ anastomoses por paciente no G-1, incluindo-se aqui, além da artéria mamária para a descendente anterior, enxertos de veia safena para outros vasos, e, no G-2, foram implantados 2,6 \pm 1,3 stents por paciente, incluindo-se os da ACDA, dos quais 50\% eram endopróteses convencionais (sem fármaco) e não foram usadas para tratar a ACDA. O maior número de vasos revascularizados no G-1 decorreu de dois motivos principais: contingente superior de pacientes com doença coronária multiarterial e facilidade técnica em se obter revascularização mais completa. Nos pacientes multiarteriais do G-1, foram obtidos $91 \%$ de revascularização completa e nos pacientes do G-2, 71\% ( $p<0,05)$.

O tempo de uso do clopidogrel $(75 \mathrm{mg} / \mathrm{dia})$ associado à aspirina, no G-2, ao lado de outras medicações específicas, variou de três a seis meses (média de $4 \pm 2$ ). Vale mencionar que 21 pacientes fizeram uso do clopidogrel por um período maior que um ano, seguindo a orientação empírica do cardiologista clínico, naquela ocasião, sem ter sido, necessariamente, uma estratégia para evitar a trombose, uma vez que não havia dados informativos suficientes a esse respeito àquela época.

A análise estatística foi realizada pelo teste de Wilcoxon, para avaliar a freqüência dos eventos após os procedimentos, e pelo teste de Mann-Whitney, para estudar a freqüência dos eventos cardíacos maiores entre os grupos abalizados. Por sua vez, utilizou-se o teste do qui-quadrado para estudar as diferenças da freqüência das variáveis categóricas entre G-1 e G-2. Quando foram observadas as restrições de Cochran, o teste aplicado foi o exato de Fischer. Em todos os testes, o nível de rejeição da hipótese de nulidade foi fixado em 0,05 ou $5 \%(p<0,05)$.

\section{RESULTADOS}

A permeabilidade da MIE anastomosada à ACDA e da ACDA que recebeu o SEF foi alta, com leve vantagem em favor da MIE, no entanto sem significância estatística (Figura 2). No G-1, dois pacientes exibiam a MIE ocluída, um no primeiro ano e outro em fase mais tardia, aos 32 meses. Acreditamos que, no primeiro caso, tenha sido por defeito técnico da sutura da MIE, pois a ACDA encontrava-se pérvia e com lesão obstrutiva grave em seu terço proximal; quanto

TABELA 1

Características demográficas dos pacientes por ocasião da indicação de ambos os procedimentos de revascularização

\begin{tabular}{|c|c|c|c|}
\hline Variáveis clínicas e anatômicas & G-1 (n = 150) & $\mathrm{G}-2(n=150)$ & Valor de $p$ \\
\hline Idade (anos) & $67 \pm 12$ & $66 \pm 11$ & NS \\
\hline Sexo feminino (\%) & 40,0 & 43,3 & NS \\
\hline Diabetes (\%) & 29,3 & 20,0 & $<0,05$ \\
\hline Hipertensão (\%) & 39,3 & 44,0 & NS \\
\hline Dislipidemia (\%) & 46,6 & 47,3 & NS \\
\hline Infarto prévio (\%) & 33,3 & 36,6 & NS \\
\hline Tabagismo (\%) & 27,3 & 29,3 & NS \\
\hline Obesidade abdominal (\%) & 40,0 & 44,0 & NS \\
\hline Angina estável (\%) & 36,6 & 39,3 & NS \\
\hline Angina instável (\%) & 27,3 & 20,0 & NS \\
\hline Infarto do miocárdio não ST (\%) & 20,0 & 19,3 & NS \\
\hline Insuficiência cardíaca (\%) & 19,3 & 14,6 & NS \\
\hline Insuficiência renal crônica (\%) & 13,3 & 12,0 & NS \\
\hline Doença vascular periférica (\%) & 27,3 & 25,3 & NS \\
\hline DPOC (\%) & 12,0 & 11,3 & NS \\
\hline Uniarterial (\%) & 13,3 & 30,6 & $<0,05$ \\
\hline Biarterial (\%) & 36,6 & 40,0 & NS \\
\hline Triarterial (\%) & 50,0 & 30,6 & $<0,001$ \\
\hline FEVE < 40\% (\%) & 36,6 & 29,3 & NS \\
\hline
\end{tabular}


Pimentel Filho WA, et al. Lesão no Segmento Proximal da Artéria Coronária Descendente Anterior: Resultados entre o Tratamento Cirúrgico (Mamária) e o Percutâneo (Stent com Fármaco). Rev Bras Cardiol Invas 2007; 15(4): 370-377.

ao segundo paciente, é provável que tenha ocorrido progressão da doença aterosclerótica justa-anastomose, já que ambas as artérias (MIE e ACDA) se encontravam ocluídas.

Já no G-2, não foram encontradas as características típicas de reestenose binária e, em quatro pacientes, a impermeabilidade da ACDA aconteceu por provável trombose da endoprótese (três com sirolimo e um com paclitaxel): um no segundo mês pelo histórico de infarto em parede anterior (fase tardia) e os demais, aos dois anos. Estes últimos três pacientes, portanto, denominando-se de trombose do SEF em fase muito tardia, de acordo com a classificação do Academic Research Consortium ${ }^{14}$. Vale salientar que dois pacientes do G-2 eram diabéticos, três deles tinham lesões excêntricas, de extensão maior que 25 $\mathrm{mm}$ e calcificadas (em dois de forma acentuada), e um paciente tinha lesão de bifurcação verdadeira ou, na classificação de Medina ${ }^{15}, 1,1,1$ (Figura 3). Quanto ao paciente que sofreu infarto no decorrer do segundo mês, o clopidogrel foi suspenso de maneira "indevida" (receio de sangramento), antes do terceiro mês, pela necessidade de intervenção dentária (extração cirúrgi-

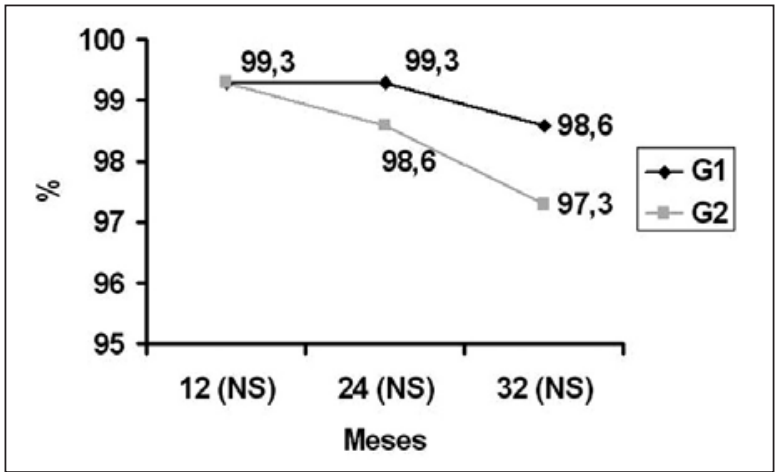

Figura 2 - Comparação entre os grupos G-1 e G-2 quanto ao porcentual de vasos (artérias mamária interna esquerda e coronária descendente anterior) que se encontravam permeáveis durante os períodos analisados.

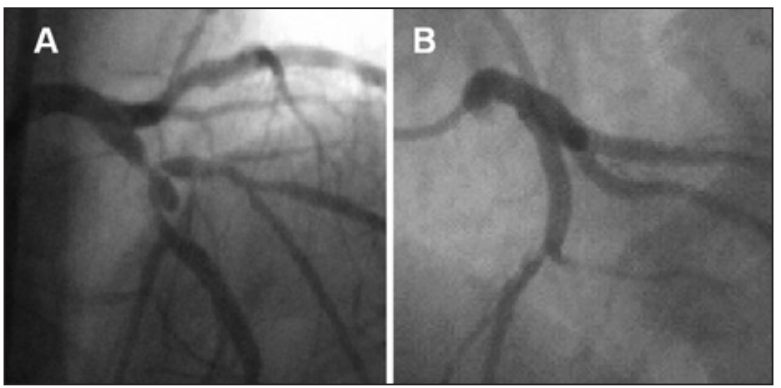

Figura 3 - A. Lesão de bifurcação verdadeira entre a artéria descendente anterior e seu primeiro ramo diagonal em paciente diabético, tratada com sucesso com dois stents, ambos com eluição de sirolimo, e utilizando a técnica de crushing com kissing-balloon final. B. Trombose muito tardia de ambos os vasos. ca). Assim sendo, nesses quatro pacientes as intervenções se caracterizaram por implantes de SEF fora dos padrões de recomendações das diversas sociedades (internacionais e nacional), incluindo as da Sociedade Brasileira de Hemodinâmica e Cardiologia Intervencionista $(\mathrm{SBHCl})$ e do Food and Drug Administration $(\mathrm{FDA})^{14}$, com denominação na língua inglesa de lesões off-labe/ ${ }^{14}$. Os demais pacientes do G-2 eram portadores de lesões no terço proximal da ACDA, enquadradas nas recomendações das diversas sociedades internacionais, da $\mathrm{SBHCl}$ e do FDA, com denominação na língua inglesa de lesões on-label (Figura 4).

Quanto aos ECAM analisados, não ocorreram diferenças entre os grupos nos três períodos (12, 24 e 32 meses), novamente com leve vantagem para o G-1 aos 32 meses, sem, entretanto, representar diferença estatisticamente significativa (Figura 5).
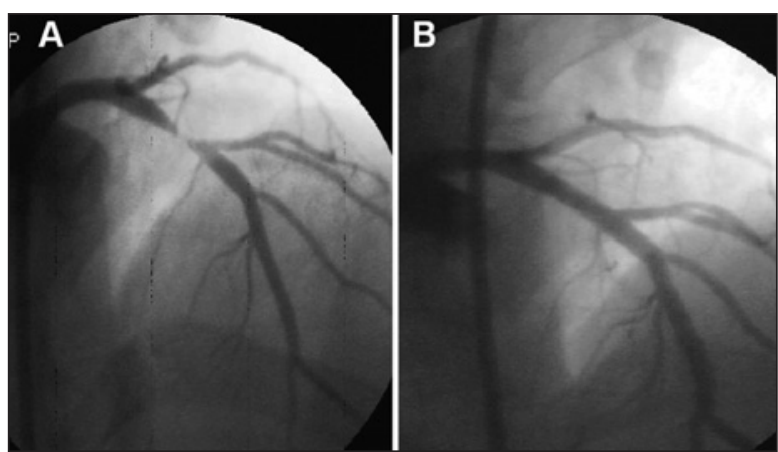

Figura 4 - A. Um dos casos deste estudo com lesão focal e de baixa complexidade localizada no terço proximal da artéria coronária descendente anterior (ACDA) em paciente do sexo feminino, sem diabetes, tratada com stent com eluição de paclitaxel. B. Reestudo 24 meses após o procedimento pelo retorno da angina (progressão da doença na artéria coronária circunflexa e reestenose de stent convencional posicionado no terço médio da artéria coronária direita), evidenciando a manutenção do resultado inicial do farmacológico na ACDA.

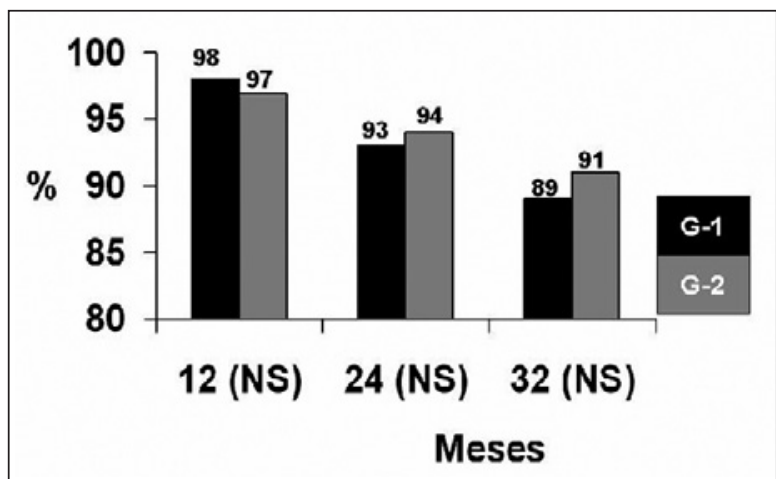

Figura 5 - Distribuição dos casos nos dois grupos estudados em diversos períodos da avaliação dos filmes, comparando os eventos cardiovasculares adversos maiores (exceto do óbito), não evidenciando diferenças estatísticas em nenhum desses segmentos de tempo apreciados. 
Pimentel Filho WA, et al. Lesão no Segmento Proximal da Artéria Coronária Descendente Anterior: Resultados entre o Tratamento Cirúrgico (Mamária) e o Percutâneo (Stent com Fármaco). Rev Bras Cardiol Invas 2007; 15(4): 370-377.

As principais causas clínicas de ECAM, que ocorreram no período entre ambos os procedimentos de revascularização e o reestudo angiográfico, estão apresentadas na Tabela 2. As principais causas anatômicas desses ECAM, em mais de $90 \%$ dos casos, foram: no G-1, a associação da oclusão de, pelo menos, um enxerto de veia safena nos pacientes multiarteriais (índice de oclusão de $25 \%$ ), principalmente nos diabéticos, juntamente com a progressão da doença aterosclerótica coronária; no G-2, a associação da revascularização incompleta e/ou reestenose de, pelo menos, um stent convencional nos pacientes multiarteriais (índice de reestenose de 33\%), preferencialmente nos diabéticos, ao lado da progressão da doença aterosclerótica coronária. Naturalmente, as oclusões dos enxertos das artérias MIE no G-1 e as oclusões dos stents no G-2 fizeram parte desses ECAM para efeito do cálculo global.

A necessidade de nova revascularização dos pacientes nesse período de avaliação, seja cirúrgica ou percutânea, está discriminada na Tabela 2.

\section{DISCUSSÃO}

Na década de 1990, a introdução do stent coronário na intervenção percutânea minimizou, de forma significativa, suas complicações agudas e os aspectos negativos da reestenose, que aconteciam quando se utilizava apenas o balão ${ }^{16}$. Portanto, reduziu significativamente a vantagem da cirurgia de revascularização coronária, mesmo na presença de lesão obstrutiva na $\operatorname{ACDA}^{10,17,18}$. Assim, apesar de ainda permanecer a necessidade de nova angioplastia quando da utilização do stent metálico, denominado hoje de convencional, já não havia diferença quanto à incidência de infarto e de óbito, tanto na fase inicial como na tardia da evolução clínica desses pacientes ${ }^{10,17-19}$.

No início dos anos 2000, apareceu uma nova família de stents com eluição de fármacos inibidores de hiperplasia, que revolucionou de maneira significativa a intervenção percutânea coronária ${ }^{20}$.

Até então, tudo indicava que o sonho da eliminação "definitiva" da reestenose tinha finalmente se realizado, sendo, naquela ocasião, motivo de especulação precipitada ${ }^{21}$. Na realidade, mesmo em se tratando de um estudo randomizado ${ }^{20}$, o número inconsistente de casos e a seleção rigorosa dos pacientes, privilegiando aqueles com lesões coronárias menos complexas, não eram suficientes para uma opinião científica definitiva, considerando a medicina baseada em evidências.

Logo se constatou que esse acontecimento, a reestenose, mantinha-se presente ${ }^{22,23}$, embora com incidência significativamente inferior, num plano sem precedentes comparativamente à obtida pelos stents convencionais ${ }^{24}$.

Nunca é demais lembrar que, já em 1986, em estudo clínico consistente ${ }^{25}$, a ACDA, quando comprometida de maneira significativa em seu segmento proximal, representava um aspecto negativo na evolução clínica quando o paciente era tratado clinicamente, ou seja, a mortalidade era significativamente maior. Quando esse paciente apresentava doença arterial coronária multivascular, essa diferença tornava-se ainda mais marcante ${ }^{25}$.

Assim, os estudos mais recentes ${ }^{11-13}$ evidenciam que, nessa circunstância, a revascularização da ACDA proporciona evolução clínica mais favorável, ou seja, não se deve adotar conduta contemplativa. Dessa forma, não é profícuo se adequar à orientação de um estudo recentemente publicado ${ }^{26}$, que analisa os pacientes de maneira generalizada.

Por outro lado, os cirurgiões têm procurado, de forma competitiva, tornar a intervenção cirúrgica menos invasiva, não usando circulação extracorpórea quando possível e realizando incisões menores (mini-

TABELA 2

Eventos cardiovasculares adversos maiores em período compreendido entre o procedimento de revascularização e o reestudo angiográfico

\begin{tabular}{lccc}
\hline ECAM (\%) & G-1 & G-2 & Valor de $\mathbf{p}$ \\
\hline Retorno da angina & 51,3 & 52,6 & NS \\
Teste provocativo de isquemia positivo & 64,0 & 66,6 & NS \\
Novo procedimento & & & NS \\
$\quad$ Cirúrgico & 6,6 & 8,0 & NS \\
$\quad$ Percutâneo & 20,6 & 22,0 & NS \\
Infarto do miocárdio & 16,6 & 18,6 & NS \\
Acidente vascular cerebral & 12,6 & 10,0 & NS \\
Associações de três itens & 72,6 & 76,6 & \\
\hline
\end{tabular}

$\mathrm{ECAM}=$ eventos cardiovasculares adversos maiores; NS = não-significante. 
toracotomia $^{27-29}$. Já há diversos relatos na literatura ${ }^{28,29}$, provenientes de grupos cirúrgicos, comparando a cirurgia minimamente invasiva com o uso do SEF no tratamento da ACDA. Os resultados, na maioria das vezes, privilegiam a intervenção cirúrgica pela menor incidência de ECAM no decorrer da evolução clínica. No entanto, nem sempre é relatado o status de cada grupo intervencionista envolvido (cirúrgico e/ou percutâneo), com seus resultados individualizados dificultando, assim, uma avaliação mais adequada desses estudos comparativos.

Neste estudo, foram notados diversos aspectos interessantes: levando-se em conta que a sobrevida dos pacientes é a finalidade primordial em qualquer forma de tratamento, seja clínico seja intervencionista (cirúrgico ou percutâneo), os pacientes submetidos a cirurgia (G-1) ou a tratamento percutâneo (G-2), a despeito da incidência relativamente baixa de ECAM em ambos os grupos no período de avaliação deste estudo, a alta permeabilidade do enxerto da MIE e do SEF para a ACDA deve ter sido o principal fator de sobrevivência a longo prazo, mesmo considerando uma população expressiva de pacientes portadores de grande complexidade clínica e com lesões multiarteriais. Também, durante esse tempo de avaliação, ocorreram falência de enxertos venosos (G-1) e incidência de reestenose (G-2), fatores esses que contribuem para uma evolução clínica menos favorável.

Outro aspecto observado foi que, em portadores de lesões complexas no terço proximal da ACDA, as denominadas off-label, principalmente nos doentes com diabetes melito do tipo 2, essa primeira geração de SEF não pareceu proporcionar o mesmo resultado favorável oferecido pela cirurgia. Nesse mesmo tempo, a cirurgia apontava a possibilidade de revascularização mais completa no paciente com doença coronária multiarterial.

Para os doentes tratados com a endoprótese, de acordo com os achados deste estudo, o clopidogrel deveria ser prescrito pelo período de um ano ou mais, quando se tratasse de portadores de lesões desfavoráveis, tipo off-label, com indicações clínicas especiais (cirurgia cardíaca contra-indicada), enquanto a aspirina deveria ser usada por tempo indefinido, desde que não houvesse contra-indicação absoluta.

O emprego dessa endoprótese na ACDA, segundo nossos dados, não seria conveniente em paciente com possibilidade de procedimento que necessitasse da suspensão do clopidogrel em período inferior a 12 meses, particularmente naqueles com lesões off-label.

Em ambos os grupos ocorreu significativa progressão da doença coronária, o que foi um fator negativo importante no prognóstico clínico dos pacientes pelo retorno de sintomas e sinais de isquemia, ressaltando, assim, a importância do tratamento clínico "agressivo" após qualquer intervenção invasiva.
Outro fator circunstancial, em nossos serviços, é o fato de que na maioria das vezes há limitação do uso de múltiplos SEF no paciente multiarterial. Assim, nesse grupo, quando do envolvimento do terço proximal da ACDA, e quando factível, sempre privilegiamos essa artéria com o SEF e, com freqüência, utilizamos $\mathrm{o}(\mathrm{s})$ stent(s) convencional(is) para os demais vasos.

Entretanto, considerando esse tempo de observação, no G-1, a falência dos enxertos venosos e, no G-2, a incidência de reestenose nos stents convencionais, pensamos que ambos os grupos merecem a reavaliação das condutas, optando, talvez, pelo uso mais freqüente dos enxertos arteriais no primeiro e dos SEF no segundo.

A presença da trombose muito tardia, vista em nossos casos submetidos a implante dos SEF de primeira geração, é uma realidade em casos com lesões mais complexas, de acordo, portanto, com a literatura atual $^{30}$.

\section{Limitações do estudo}

Este estudo apresenta diversas limitações: 1. foi realizado de maneira retrospectiva e apenas com a participação de dois centros interligados; 2. os pacientes foram avaliados predominantemente pela informação clínica das equipes envolvidas ou por contato telefônico com o médico cardiologista; 3. os integrantes do G-1 apresentavam maior índice de diabetes melito e de doença multiarterial, o que dificulta uma análise comparativa mais real; 4. foi excluído, dos ECAM, o porcentual de óbitos em ambos os grupos dada a característica particular deste estudo, impossibilitando, portanto, uma opinião mais abalizada sobre esse aspecto clínico.

Finalmente, acreditamos que, apesar das referidas limitações, o estudo oferece a oportunidade de analisar e comparar, a longo prazo, clínica e angiograficamente, os dados pertinentes a essa nossa avaliação de acordo com as técnicas intervencionistas estudadas.

\section{CONCLUSÃO}

Em nosso estudo, foi de grande relevância clínica a permeabilidade dos vasos, tanto da MIE como da ACDA que recebeu o SEF, independentemente do comprometimento dos enxertos venosos no G-1 e da reestenose no G-2.

A progressão da doença coronária foi evidente, demonstrando, portanto, a necessidade de tratamento clínico efetivo após esses procedimentos.

No momento, a responsabilidade do SEF é inquestionavelmente enorme naqueles casos de maior complexidade da lesão tipo off-label localizada no terço proximal na ACDA, diante dos resultados cirúrgicos ainda superiores quando do uso do enxerto com a MIE. 
Pimentel Filho WA, et al. Lesão no Segmento Proximal da Artéria Coronária Descendente Anterior: Resultados entre o Tratamento Cirúrgico (Mamária) e o Percutâneo (Stent com Fármaco). Rev Bras Cardiol Invas 2007; 15(4): 370-377.

Sempre personalizando a situação clínica de cada paciente, em presença de lesão obstrutiva grave no terço proximal da ACDA, principalmente naqueles doentes sintomáticos e/ou com grandes áreas de isquemia e sem circulação colateral visível direcionada para esse vaso, o cardiologista deve avaliar com grande responsabilidade, baseado em evidências científicas, qual a intervenção médica mais adequada.

Enfim, a nosso ver, em futuro próximo, tanto nos pacientes uni como nos multiarteriais com comprometimento do terço proximal da ACDA, diabéticos ou não, há que se comparar, de forma randomizada, o(s) enxerto(s) arterial(is) realizado(s) na cirurgia com as "novas gerações" de SEF, a fim de se obter segurança no emprego dessas endopróteses de maneira mais abrangente.

\section{REFERÊNCIAS BIBLIOGRÁFICAS}

1. Barner HB, Swartz MT, Mudd JG, Tyras DH. Late patency of the internal mammary artery as a coronary bypass conduit. Ann Thorac Surg. 1982;34(4):408-12.

2. Loop FD, Lytle BW, Cosgrove DM, Stewart RM, Goormastic $M$, Williams GW, et al. Influence of internal-mammaryartery graft on 10-year survival and other cardiac events. $\mathrm{N}$ Engl J Med. 1986;314(1):1-6.

3. Hannan EL, Racz MJ, Walford G, Jones RH, Ryan TJ, Bennett $\mathrm{E}$, et al. Long-term outcomes of coronary-artery bypass grafting versus stent implantation. $N$ Engl J Med. 2005;352(21):2174-83.

4. King SB $3^{\text {rd }}$. Is surgery preferred for the diabetic with multivessel disease? Circulation. 2005;112(10):1500-7.

5. Berry C, Tardif JC, Bourassa MG. Coronary heart disease in patients with diabetes: part II: recent advances in coronary revascularization. J Am Coll Cardiol. 2007;49(6):643-56.

6. Stankovic G, Cosgrave J, Chieffo A, lakovou I, Sangiorgi G, Montorfano M, et al. Impact of sirolimus-eluting and paclitaxeleluting stents on outcome in patients with diabetes mellitus and stenting in more than one coronary artery. Am J Cardiol. 2006;98(3):362-6.

7. Smith SC Jr, Feldman TE, Hirshfeld JW Jr, Jacobs AK, Kern MJ, King SB 3rd, et al. ACC/AHA/SCAI 2005 Guideline Update for Percutaneous Coronary Intervention-Summary Article: a report of the American College of Cardiology/ American Heart Association Task Force on Practice Guidelines (ACC/AHA/SCAI Writing Committee to Update the 2001 Guidelines for Percutaneous Coronary Intervention). J Am Coll Cardiol. 2006;47(1):216-35.

8. Boyden TF, Nallamothu BK, Moscucci M, Chan PS, Grossman PM, Tsai TT, et al. Meta-analysis of randomized trials of drug-eluting stents versus bare metal stents in patients with diabetes mellitus. Am J Cardiol. 2007;99(10):1399-402.

9. Stone GW, Moses JW, Ellis SG, Schofer J, Dawkins KD, Morice MC, et al. Safety and efficacy of sirolimus- and paclitaxel-eluting coronary stents. N Engl J Med. 2007;356(10): 998-1008.

10. Sawhney N, Moses JW, Leon MB, Kuntz RE, Popma JJ, Bachinsky $W$, et al. Treatment of left anterior descending coronary artery disease with sirolimus-eluting stents. Circulation. 2004;110(4):374-9.

11. Dangas G, Ellis SG, Shlofmitz R, Katz S, Fish D, Martin S, et al. For the TAXUS-IV Investigators. Outcomes of paclitaxeleluting stent implantation in patients with stenosis of the left anterior descending coronary artery. J Am Coll Cardiol. 2005;45(8):1186-92.

12. Guardado JH, Moreno R, Costa J, Viscayno MJP, Segura L, Alfonso $\mathrm{F}$, et al. Revascularização da descendente anterior proximal com stents revestidos por fármacos. Arq Bras Cardiol. 2007;88(2):159-66.

13. Khattab AA, Hamm CW, Senges J, Toelg R, Geist V, Bonzel $\mathrm{T}$, et al. Incidence and predictors of target vessel revascularization after sirolimus-eluting stent treatment for proximal left anterior descending artery stenoses among 2274 patients fromthe prospectivemulticenter German Cypher Stent Registry. Clin Res Cardiol. 2007;96(5):279-84.

14. Cutlip DE, Windecker S, Mehran R, Boam A, Cohen DJ, van Es GA, et al. On behalf of the Academic Research Consortium. Clinical end points in coronary stents trials: a case for standardized definitions. Circulation. 2007;115(17):2344-51.

15. Legrand $V$, Thomas $M$, Zelisko $M$, Bruyne BD, Reifart N, Steigen $\mathrm{T}$, et al. Percutaneous coronary intervention of bifurcation lesions: state-of-the-art. Insights from the second meeting of the European bifurcation club. Eurointerv. 2007;3 (1):44-9.

16. BARI Investigators. The final 10-year follow-up results from the BARI randomized trial. J Am Coll Cardiol. 2007;49(15): 1600-6.

17. Serruys PW, de Jaegere P, Kiemeneij F, Macaya C, Rutsch W, Heyndrickx G. A comparison of balloon-expandablestent implantation with balloon angioplasty in patients with coronary artery disease. Benestent Study Group. N Engl J Med. 1994;331(8):489-95.

18. Fischman DL, Leon MB, Baim DS, Schatz RA, Savage MP, Penn I, et al. A randomized comparison of coronary-stent placement and balloon angioplasty in the treatment of coronary artery disease. Stent Restenosis Study Investigators. N Engl J Med. 1994;331(8):496-501.

19. Aoki J, Ong AT, Arampatzis CA, Vijaykumar M, Rodriguez Granillo GA, Disco CM, et al. Comparison of three-year outcomes after coronary stenting versus coronary artery bypass grafting in patients with multivessel coronary disease, including involvement of the left anterior descending coronary artery proximally (a subanalysis of the arterial revascularization therapies study trial). Am J Cardiol. 2004;94(5):627-31.

20. Morice MC, Serruys PW, Sousa JE, Fajadet J, Ban Hayashi E, Perin $M$, et al. RAVEL study group. A randomized comparison of a sirolimus-eluting stent with a standard stent for coronary revascularization. N Engl J Med. 2002;346(23):1772-80.

21. Regar E, Serruys PW. The Ravel trial. Zero percent restenosis: a cardiologists dream comes true! Rev Esp Cardiol. 2002;55 (5):459-62.

22. Lemos PA, Saia F, Ligthart JM, Arampatzis CA, Sianos G, Tanabe $\mathrm{K}$, et al. Coronary restenosis after sirolimus-eluting stent implantation: morphological description and mechanistic analysis from a consecutive series of cases. Circulation. 2003;108(3):257-60.

23. Popma JJ, Leon MB, Moses JW, Holmes DR Jr, Cox N, Fitzpatrick $M$, et al. SIRIUS Investigators. Quantitative assessment of angiographic restenosis after sirolimus-eluting stent implantation in native coronary arteries. Circulation. 2004;110(25):3773-80.

24. Serruys PW, Ong ATL, Morice MC, Bruyne BD, Colombo A, Macaya $C$, et al. Arterial revascularisation therapies study part II - sirolimus-eluting stents for the treatment of patients with multivessel de novo coronary artery lesions. Eurolnterv. $2005 ; 1: 147-56$

25. Klein LW, Weintraub WS, Agarwal JB, Schneider RM, Seelaus $\mathrm{PA}$, Katz RI, et al. Prognostic significance of severe narrow- 
Pimentel Filho WA, et al. Lesão no Segmento Proximal da Artéria Coronária Descendente Anterior: Resultados entre o Tratamento Cirúrgico (Mamária) e o Percutâneo (Stent com Fármaco). Rev Bras Cardiol Invas 2007; 15(4): 370-377.

ing of the proximal portion of the left anterior descending coronary artery. Am J Cardiol. 1986;58(1):42-6.

26. Boden WE, O'Rourke RA, Teo KK, Hartigan PM, Maron DJ, Kostuk WJ, et al. for the COURAGE Trial Research Group. Optimal medical therapy with or without $\mathrm{PCl}$ for stable coronary disease. N Engl J Med. 2007;356(15):1503-16.

27. Gauze T, Rosa FA, Salvi WF Jr., Tamazato E. Revascularização miocárdica por minitoracotomia esquerda: série de casos. Braz J Cardiovasc Surg. 2007;22(1):111-5.

28. Moshkovitz Y, Mohr R, Braunstein R, Zivi E, Uretzky G, Ben-Gal $Y$, et al. Revascularization of left anterior descending coronary artery in patients with single and multivessel disease: comparison between off-pump internal thoracic artery and drug-eluting stent. Chest. 2005;128(2):804-9.

29. Hong SJ, Lim DS, Seo HS, Kim YH, Shim WJ, Park CG, et al. Percutaneous coronary intervention with drug-eluting stent implantation $v s$ minimally invasive direct coronary artery bypass (MIDCAB) in patients with left anterior descending coronary artery stenosis. Catheter Cardiovasc Interv. 2005;64(1):75-81.

30. Lüscher TF, Steffel J, Eberli FR, Joner M, Nakazawa G, Tanner FC, et al. Drug-eluting stent and coronary thrombosis: biological mechanisms and clinical implications. Circulation. $2007 ; 115(8): 1051-8$. 